\title{
Discriminative Stimulus Effects of Pentobarbital in Rhesus Monkeys: Tests of Stimulus Generalization and Duration of Action
}

\author{
Gail Winger and Seymore Herling* \\ Departments of Pharmacology and Psychology, University of Michigan, Ann Arbor, Michigan 48109, USA
}

\begin{abstract}
Rhesus monkeys were trained to emit 20 or 30 consecutive responses on one lever following an IM injection of pentobarbital (10 or $18 \mathrm{mg} / \mathrm{kg}$ ) and the same number of consecutive responses on another lever following an injection of saline. The required number of correct consecutive responses in both cases resulted in food delivery. When responding was reliably under the control of the presession injection, the ability of a variety of other compounds to produce pentobarbital-appropriate responding was examined. Diazepam, clobazam, methohexital, pentobarbital, and phenobarbital, given 10 or $20 \mathrm{~min}$ before the session, produced dose-related pentobarbital-appropriate responding in each monkey. Ethylketazocine and dextromethorphan produced responding primarily on the saline-appropriate lever, whereas codeine, cyclazocine, dextrorphan, and ketamine resulted in responding that was, on the average, intermediate between that appropriate for pentobarbital and that appropriate for saline. When tested at various times after their injection, methohexital $(3.2 \mathrm{mg} / \mathrm{kg})$ and pentobarbital $(10 \mathrm{mg} / \mathrm{kg})$ produced pentobarbital-appropriate responding within $10 \mathrm{~min}$. Barbital $(56 \mathrm{mg} / \mathrm{kg})$ resulted in pentobarbitalappropriate responding only if at least $1 \mathrm{~h}$ intervened between the injection and the experimental session. The discriminative effects of methohexital, pentobarbital, and barbital lasted approximately $20-60,120-240$, and $480-720 \mathrm{~min}$, respectively. The time-course of the discriminative stimulus effects of barbiturates in the rhesus monkey appears to parallel closely other pharmacological actions of these compounds.
\end{abstract}

Key words: Pentobarbital - Drug discrimination - Rhesus monkeys - Barbiturates - Benzodiazepines - Duration of action

When a designated behavior is reinforced in the presence of a specified environmental event, and the rate of occurrence of the behavior increases in the presence of this event, the event is called a discriminative stimulus. Many centrally acting drugs have been shown to be very effective discriminative stimuli (Barry 1974); animals can be trained to emit one response following a drug injection, and another response if no drug has been administered. In a well-trained subject, generalization tests can be conducted by administering various other

\footnotetext{
* Present address: National Institute on Drug Abuse, Addiction Research Center, P.O. Box 12390, Lexington, KY 40583, USA Offprint requests to: $\mathrm{G}$. Winger
}

drugs and determining the extent to which responding occurs on the drug or nondrug-associated lever.

Although the discriminative stimulus properties of pentobarbital have been studied extensively in rodents (e.g., Overton 1966; Barry 1974; Krimmer 1974; Johansson and Jarbe 1975; Jarbe 1976) and to some extent in pigeons (e.g., Leberer and Fowler 1977; Jarbe and Ohlin 1979; Witkin et al. 1980; Herling et al. 1980), there have been few studies of the discriminative stimulus effects of pentobarbital in primates. Trost and Ferraro (1974) trained rhesus monkeys to make lever position choices based on whether they had received 2 or $10 \mathrm{mg} / \mathrm{kg}$ pentobarbital before the session. The monkeys were able to discriminate between these two doses, and on generalization testing, showed relatively equal choices of the two response levers at an average dose of $4 \mathrm{mg} / \mathrm{kg}$.

Tests of generalization of other drugs to a pentobarbitaltrained discriminative stimulus, however, have not been reported in the monkey. In other species, including the rat, gerbil, and pigeon, there is considerable agreement as to the drugs that generalize to pentobarbital. Appropriate doses of the barbiturates, amobarbital, barbital, phenobarbital, and methohexital, as well as the anti-anxiety agents, diazepam, chlordiazepoxide, clobazam, and meprobamate, have been shown to produce pentobarbital-like discriminative effects in several species. In contrast, drugs from other pharmacological classes (e.g., narcotics, psychomotor stimulants) generally do not produce pentobarbital-appropriate responding (e.g., Barry 1974; Herling et al. 1980).

In addition to providing information on drug classification, drug discrimination techniques can also be used to indicate the onset and duration of drug-induced interoceptive effects. Barry and Krimmer (1978a), for example, demonstrated that the discriminative effects of $10 \mathrm{mg} / \mathrm{kg}$ IP pentobarbital were present $2.5 \mathrm{~min}$ after the injection, and still present, but to a lesser degree, $60 \mathrm{~min}$ following the injection. By $120 \mathrm{~min}$, the pentobarbital discriminative effects had subsided.

The present study investigated the discriminative stimulus effects of pentobarbital in the rhesus monkey by assessing the extent to which several drugs from various pharmacological classes produced discriminative effects similar to those of pentobarbital. Among the drugs selected for study were a number of barbiturates and benzodiazepines that have been shown to generalize to pentobarbital in other species. Ketamine and dextrophan, drugs which have been shown in pigeons to produce intermediate levels of pentobarbitalappropriate responding (Herling et al. 1980), were among several non-barbiturates that were studied. Evaluation was also made of the onset and duration of the discriminative 
effects of barbiturates with different durations of pharmacological activity.

\section{Materials and Methods}

Subjects. The subjects were three adult male rhesus monkeys (Macaca mulatta) maintained at approximately $90 \%$ of their free feeding weights $(9.0-10.0 \mathrm{~kg})$. The monkeys were fed sufficient Purina High Protein Monkey Chow after each session to maintain their reduced body weight. Water $(1000 \mathrm{ml}$, twice daily) was available in their home cages and each monkey was given $40 \mathrm{mg}$ isoniazid daily on a sugar cube. Fresh fruit was provided several times per week. Each monkey had an extensive laboratory history including experience lever pressing under fixed-ratio schedules of food and intravenous cocaine or ethanol delivery. Two of the monkeys had also received chronic injections of ethylketazocine for several months. None of the monkeys, however, had received drug injections or participated in an experiment for at least 6 months prior to the start of this experiment.

Apparatus. Prior to each experimental session, the monkeys were seated in primate restraining chairs and placed in soundattenuating wooden test chambers. Each chamber was equipped with two response levers (BRS/LVE, Beltsville, MD, model PRL-001/121-07) mounted equidistant from a central food receptacle. A force of approximately $40 \mathrm{~g}$ was required to operate the levers. The excursion distance for the levers was approximately $2 \mathrm{~mm}$. The levers and food receptacle were located approximately $50 \mathrm{~cm}$ above the floor of the chamber, within reach of the monkey seated in the chair. An exhaust fan was used for ventilation, and a speaker mounted inside the chamber provided white noise to mask extraneous sounds.

The start of each session was signaled by the illumination of two 7-W blue lights mounted at the top of the front wall of the chamber. Banana-flavored food pellets $(300 \mathrm{mg}$; P. J. Noyes Co., Lancaster; NH. Formula G) were delivered to the food receptacle by a pellet dispenser (Ralph Gerbrands Co., Arlington, MA; model A) that was mounted outside the chamber. A Texas Instruments Inc. 960A computer was used to control experimental sessions, and to collect and analyze data. In addition, cumulative response recorders (Ralph Gerbrands Co.; model C-3) recorded the performance of each monkey.

Discrimination Training. Initially, a single press, or response (fixed-ratio 1; FR1), on one of the two available levers produced a food pellet. The lever on which responses resulted in food delivery alternated from one day to the next. On days when the right lever was designated correct, an IM injection of $10 \mathrm{mg} / \mathrm{kg}$ pentobarbital was given $10 \mathrm{~min}$ prior to the session. On days when responses on the left lever produced food, an IM injection of saline preceded the session. The ratio requirement was gradually increased over sessions until 20 consecutive responses (FR 20) on the appropriate lever were required for food delivery. Responses on the inappropriate lever reset the FR response requirement on the lever designated as correct for that session. Training continued at a FR 20 value, with alternate-day injections of saline or $10 \mathrm{mg} / \mathrm{kg}$ pentobarbital, until the discrimination criteria were met (see below), or until it appeared that the training conditions were not optimal for a particular monkey. One monkey (700) reached the training criteria and was maintained at a FR 20 and a training dose of $10 \mathrm{mg} / \mathrm{kg}$ pentobarbital injected $10 \mathrm{~min}$ before the session. In the other two monkeys ( 760 and 28 ), the FR requirement was increased to 30 , and the pretreatment interval was increased to $20 \mathrm{~min}$. In one of these monkeys (28), the dose of pentobarbital was increased to $18 \mathrm{mg} / \mathrm{kg}$.

Sessions were conducted 6 days per week and ended after 75 food pellet deliveries or after $1 \mathrm{~h}$, whichever occurred first. Training sessions continued until the following criteria were met: 1) no more responses than two times the fixed-ratio value (i.e., 40 or 60 responses, depending on the monkey) occurred before the first food delivery of the session, and 2) a minimum of $90 \%$ of the responses during the entire session occurred on the correct lever. Before testing was initiated, these criteria were met for five consecutive sessions in which saline and pentobarbital injections alternated and then for four consecutive sessions in which pentobarbital and saline were administered in a double alternation sequence.

Discrimination Testing. On test days, the procedure was altered so that, throughout the entire test session, 20 or 30 consecutive responses on either one of the two levers resulted in food delivery; otherwise, test sessions were identical to training sessions. Tests were first conducted with various doses of pentobarbital and its vehicle. Several drugs from various pharmacological classes were also evaluated. Test sessions typically alternated with training sessions during the week. If an animal failed to meet the training criteria, training continued until these criteria were met for at least two consecutive sessions.

Onset and Duration of the Discriminative Stimulus Effects of Various Barbiturates. Determination of the onset and duration of the discriminative effects of a number of barbiturates, using the testing procedure described above, was accomplished by administering the drug at varying times before the session. The drugs, doses and pretreatment times evaluated were methohexital, $3.2 \mathrm{mg} / \mathrm{kg}$, at $1,10,20,60$, and $120 \mathrm{~min}$; pentobarbital, $10 \mathrm{mg} / \mathrm{kg}$, at $1,10,20,60,120$, and $240 \mathrm{~min}$; and barbital, $56 \mathrm{mg} / \mathrm{kg}$, at 20, 60, 120, 240, 480, 720, 960 , and $1440 \mathrm{~min}$. These drugs were selected because they are pharmacologically similar, differing primarily in their durations of action (Sharpless 1970). The doses of methohexital and pentobarbital selected were the lowest doses that produced $100 \%$ pentobarbital-appropriate responding when given 10 or $20 \mathrm{~min}$ before the session. The dose of barbital was selected on the basis of its potency relative to pentobarbital and methohexital as determined in other behavioral tests (Winger et al. 1975). When evaluating pretreatment times of more than $1 \mathrm{~h}$, the monkeys were injected with the drug and returned to their home cages. Approximately $20 \mathrm{~min}$ before the pretreatment interval elapsed, the monkeys were rechaired, injected with saline, and placed in the experimental chamber. The session began 20 min later. For pretreatments of less than $1 \mathrm{~h}$, the monkeys were placed in the chamber immediately following the injection, and the session started at the designated time.

Drugs. Sodium pentobarbital, sodium phenobarbital, sodium barbital, sodium methohexital, ketamine hydrochloride, and codeine phosphate were obtained commercially. Dextrophan tartrate, dextromethorphan hydrobromide, and diazepam were gifts from Hoffmann-La Roche, Inc., Nutley, NJ. 


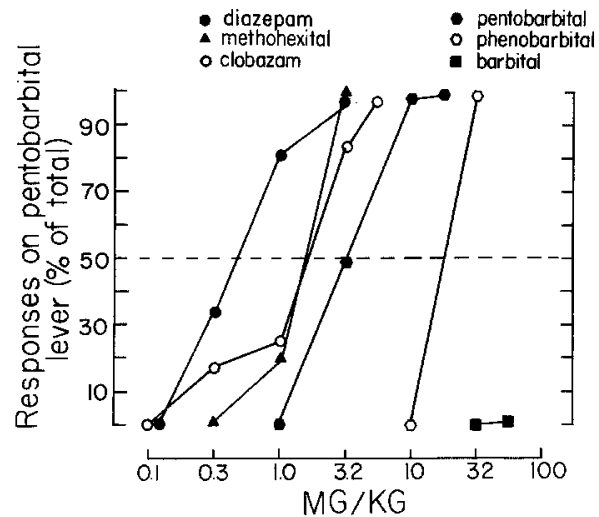

Fig. 1. Dose-response curves for the discriminative stimulus effects of pentobarbital, four drugs that produced effects similar to those of pentobarbital, and one drug that did not produce these effects when tested 10 or $20 \mathrm{~min}$ after IM injection. Ordinates are the average number of responses made on the pentobarbital-appropriate lever, expressed as a percent of the total session responses. The abscissae are the doses of the drugs in $\mathrm{mg} / \mathrm{kg}$. Each point represents the mean of one observation in each of three monkeys

Clobazam was provided by Hoechst-Roussel Pharmaceuticals, Inc., Somerville, NJ. Ethylketazocine methane sulfonate and cyclazocine base were provided by Dr. W. Michne, Sterling-Winthrop Research Institute, Rensselaer, NY.

The vehicle for pentobarbital was a solution containing ethanol, propylene glycol, and sterile water, in a ratio of $1: 2: 7$. Diazepam and clobazam were dissolved in a solution containing $40 \%$ propylene glycol, $10 \%$ ethanol, $5 \%$ sodium benzoate and benzoic acid, $1.5 \%$ benzyl alcohol, and $43.5 \%$ sterile water. Cyclazocine and ethylketazocine were dissolved in sterile water to which a small amount of lactic acid was added; sodium hydroxide was used to adjust the $\mathrm{pH}$ of the solution to above 4. Dextromethorphan, dextrorphan, codeine, and ketamine were dissolved in $0.9 \%$ sterile saline; methohexital, phenobarbital, and barbital were dissolved in sterile water. Injections of all drugs were made into the muscle of the thigh, usually in a volume of $0.1 \mathrm{ml} / \mathrm{kg}$. Drug doses refer to the forms described above.

Data Analysis. The data for test sessions are presented as the average number of responses that were made on the pentobarbital-appropriate lever throughout the session, expressed as a percentage of the total number of responses. A test drug was considered to produce discriminative effects similar to the training dose of pentobarbital if at least $90 \%$ of the total session responses were emitted on the pentobarbitalappropriate lever.

\section{Results}

Drugs that Produced Discriminative Effects Similar to those of Pentobarbital. All three monkeys acquired the pentobarbitalsaline discrimination. The number of sessions required to reach criterion performance was 36,54 , and 84 for monkeys 700,760 , and 28 , respectively. When the pentobarbital vehicle was administered before a test session, each monkey responded exclusively on the saline-appropriate lever. Drugs that at some dose produced at least $90 \%$ pentobarbitalappropriate responding in each monkey are shown in Fig. 1.
Table 1. Percentage of total responses on pentobarbital-appropriate lever produced by non-barbiturates in individual monkeys

\begin{tabular}{lllll}
\hline Drug $\begin{array}{l}\text { Maximum pentobarbital } \\
\text { (dose range tested, } \mathrm{mg} / \mathrm{kg})\end{array}$ & \multicolumn{3}{l}{$\begin{array}{l}\text { Maxponse. } \\
\text { responkey no. }\end{array}$} \\
\cline { 3 - 5 } & & 760 & 700 & 28 \\
\hline Cyclazocine & $(0.001-0.1)$ & $100(0.01)^{\mathrm{a}}$ & $0(-)$ & $1(0.01)$ \\
Dextrorphan & $(1.0-5.6)$ & $33(1.0)$ & $90(3.2)$ & $2(5.6)$ \\
Ketamine & $(0.3-3.2)$ & $100(1.0)$ & $65(3.2)$ & $1(1.8)$ \\
Codeine & $(0.3-5.6)$ & $17(3.2)$ & $41(1.8)$ & $75(5.6)$ \\
Dextromethorphan & $(0.3-3.2)$ & $0(-)$ & $0(-)$ & $6(1.8)$ \\
Ethylketazocine & $(0.001-0.03)$ & $4(0.01)$ & $13(0.01)$ & $0(-)$
\end{tabular}

a Given in parentheses is the dose of drug producing the maximum pentobarbital response

The lowest doses of these drugs produced predominantly saline-appropriate responding. As the dose was increased, the percentage of pentobarbital-appropriate responding produced by each drug, except barbital, increased. The lowest doses needed to produce $90 \%$ or more pentobarbitalappropriate responding were $3.2 \mathrm{mg} / \mathrm{kg}$ methohexital, $10 \mathrm{mg} / \mathrm{kg}$ pentobarbital, $32 \mathrm{mg} / \mathrm{kg}$ phenobarbital, $3.2 \mathrm{mg} / \mathrm{kg}$ diazepam, and $5.6 \mathrm{mg} / \mathrm{kg}$ clobazam. These doses of these drugs had little or no effect on the rate of responding. Barbital ( 32 or $56 \mathrm{mg} / \mathrm{kg}$ ), administered 10 or $20 \mathrm{~min}$ before the session, produced only saline-appropriate responding.

Drugs that Failed to Produce Discriminative Effects Similar to those of Pentobarbital. Data for drugs that did not produce $90 \%$ or more pentobarbital-appropriate responding in all monkeys are shown in Table 1. Each drug was evaluated in a range of doses, the largest of which produced marked decreases in the rate of responding. Ethylketazocine and dextromethorphan resulted in $13 \%$ or less pentobarbitalappropriate responding in each monkey. Codeine, however, produced responding that was, in each monkey, intermediate between that appropriate for saline and that appropriate for pentobarbital. Dextrorphan, ketamine, and cyclazocine each produced over $90 \%$ responding on the pentobarbitalappropriate lever in one of the three monkeys and considerably less pentobarbital-appropriate responding in the other two. Monkey 28 responded primarily on the salineappropriate lever following the administration of each of these drugs; monkey 700 made $90 \%$ pentobarbitalappropriate responses following the administration of an appropriate dose of dextrorphan; and monkey 760 made $100 \%$ pentobarbital-lever responses following the administration of appropriate doses of cyclazocine and ketamine.

The data shown in Table 1 show the maximum percent of responding on the pentobarbital-appropriate lever produced by the various drugs. Only slightly less responding on the pentobarbital-appropriate lever occurred at either higher or lower doses of dextrorphan, ketamine, or cyclazocine. In the case of codeine, however, the dose producing the maximum percent of pentobarbital-appropriate responding was the highest dose evaluated in each monkey, and these doses produced considerable suppression of rates of responding. Doses lower than these doses of codeine produced little or no drug-appropriate responding in any of the monkeys. 


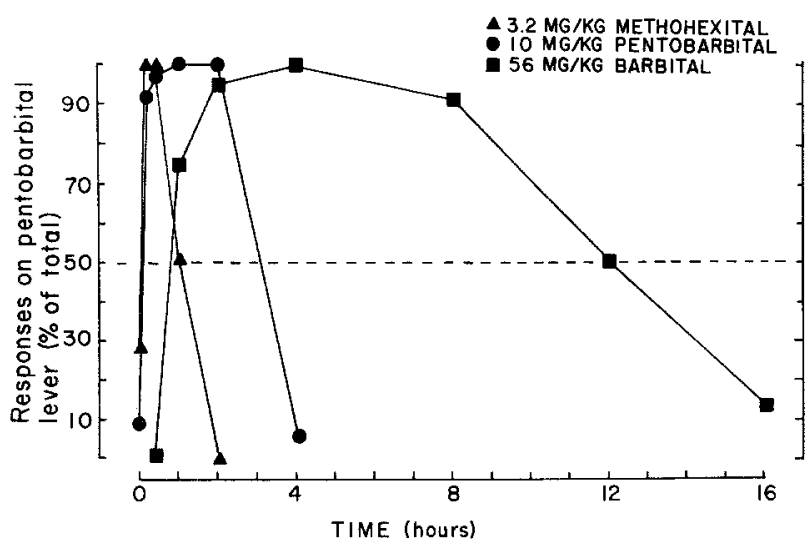

Fig. 2. Onset and duration of the discriminative stimulus effects of methohexital, pentobarbital, and barbital. Ordinates are the average number of responses made on the pentobarbital-appropriate lever, expressed as percent of the total session responses. The abscissae are the hours following the injection of the indicated dose of each drug. Each point represents the mean of one observation in each of three monkeys except for the points at $1 \mathrm{~min}$ and $20 \mathrm{~min}$ for methohexital and pentobarbital. These are means of one observation in each of two monkeys

Onset and Duration of the Discriminative Stimulus Effects of Barbiturates. The mean onset and duration of the discriminative stimulus effects of three barbiturates are shown in Fig. 2. One minute after their injection, $3.2 \mathrm{mg} / \mathrm{kg}$ methohexital and $10 \mathrm{mg} / \mathrm{kg}$ pentobarbital produced some pentobarbital-appropriate responding in monkey 760 and only saline-appropriate responding in monkey 28. Monkey 700 was not tested at this time interval. Ten minutes after their injection, these doses of methohexital and pentobarbital produced primarily pentobarbital-appropriate responding in all three monkeys. Barbital $(56 \mathrm{mg} / \mathrm{kg})$ did not produce pentobarbital-appropriate responding until $60 \mathrm{~min}$ after its injection in two monkeys and $120 \mathrm{~min}$ after its injection in the third.

The duration of the discriminative stimulus effects of the three drugs differed markedly (Fig. 2). Methohexital $(3.2 \mathrm{mg} / \mathrm{kg})$ no longer produced pentobarbital-appropriate responding in any of the monkeys $2 \mathrm{~h}$ after its injection. Pentobarbital $(10 \mathrm{mg} / \mathrm{kg})$ produced over $99 \%$ pentobarbitalappropriate responding at 60 and $120 \mathrm{~min}$ after the injection, but only $9 \%$ or less at $240 \mathrm{~min}$. The discriminative effects of $56 \mathrm{mg} / \mathrm{kg}$ barbital decreased more gradually and the duration of the effect was more prolonged. In each monkey, $100 \%$ pentobarbital-appropriate responding occurred $240 \mathrm{~min}$ after the injection of $56 \mathrm{mg} / \mathrm{kg}$ barbital, and in one monkey (760) declined slowly to $0 \%$ at $1440 \mathrm{~min}$. In the other two monkeys, the loss of stimulus control by barbital was more abrupt, falling from 100 to $0 \%$ pentobarbital-appropriate responding between either 720 and $960 \mathrm{~min}$ (monkey 700 ) or 480 and $720 \mathrm{~min}$ (monkey 28) after the injection.

\section{Discussion}

Each of the rhesus monkeys trained to discriminate pentobarbital from saline in the present experiment emitted pentobarbital-appropriate responses following injections of pentobarbital, methohexital, phenobarbital, diazepam, clobazam, and, if administered $1-2 \mathrm{~h}$ before the session, barbital. These data showing generalization of various barbiturates to pentobarbital in rhesus monkeys are similar to those obtained previously in rodents and pigeons (Overton 1966; Barry 1974; Jarbe 1976; Herling et al. 1980). In addition, the similarity between the discriminative effects of barbiturates and benzodiazepines seen in the present study has also been observed in other species (Barry 1974; Colpaert et al. 1976; Herling et al. 1980). Although a discrimination between pentobarbital and the benzodiazepine chlordiazepoxide can be established (e.g., Krimmer and Barry 1979), and differences between the discriminative effects of these drugs can be demonstrated (e.g., Overton 1966; Barry and Krimmer 1977), there appears to be sufficient similarity between the discriminative stimulus effects of barbiturates and benzodiazepines that animals trained to discriminate a barbiturate from saline make drug-appropriate responses following the administration of benzodiazepines, and vice versa (Colpaert et al. 1976; Jarbe 1976; Herling et al. 1980). The relative potencies of these compounds in producing barbiturate- or benzodiazepine-like discriminative effects is remarkably consistent across species (cf. Fig. 1; Colpaert et al. 1976; Herling et al. 1980).

The onset and duration of discriminative stimulus effects varied among the different barbiturates tested. Methohexital, administered IM, had a rapid onset and relatively short duration of action as a discriminative stimulus. In the monkey, methohexital, given IV in doses of $4-6 \mathrm{mg} / \mathrm{kg}$, produces anesthesia for about $5 \mathrm{~min}$ (Winger unpublished observations). Under these conditions, its action is terminated primarily by rapid redistribution from the brain to other tissues, followed by relatively rapid metabolic degradation (Breimer 1976). Redistribution as a mechanism of termination should be less important following IM administration, since using this route the drug does not travel as a bolus to the brain and its duration of action would be determined primarily by its rate of metabolic breakdown.

Pentobarbital administered IM, like methohexital, had a rapid onset, whereas its duration as a discriminative stimulus was between 2 and $4 \mathrm{~h}$, approximately twice that of methohexital. Pentobarbital's action is terminated primarily by metabolism following both IV and IM administration. The duration of discriminative effect following IM injection of pentobarbital and methohexital in the present study, therefore, may be indicative of the relative metabolic rates of these two compounds, being less confounded with termination through redistribution.

Barbital, a drug that is poorly lipid soluble, required at least $1 \mathrm{~h}$ before its discriminative effects were apparent. Barbital is not metabolized to any great extent, but is excreted unchanged, resulting in a relatively long duration of action as a discriminative stimulus, approximately four times that of pentobarbital. Generally, the present data on the onset and duration of the discriminative effects of these barbiturates closely parallel other pharmacological properties of these drugs (e.g., hypnosis, sedation, anesthesia).

The two narcotics, ethylketazocine and codeine, generated predominately saline-appropriate responding, although responses on both levers were produced by the highest dose of codeine that was tested in each monkey. These results are generally consistent with results obtained in rhesus monkeys trained to discriminate either ethylketazocine (Hein et al. 1981) or codeine (unpublished observations) from saline, in that barbiturates (methohexital, pentobarbital) produce little or no drug-appropriate responding in these monkeys. 
In contrast to the effects that were observed for most of the drugs in the present study, the effects of ketamine, dextrorphan, and cyclazocine were more variable. For example, ketamine produced exclusively saline-lever responses in one monkey, dose-related pentobarbital-appropriate responding in a second monkey, and responses on both levers in a third. A similar result has been described in rats and pigeons (Overton 1975; Herling et al. 1980). That ketamine, dextrorphan, and cyclazocine may have similar effects accounting for the type of variability observed in the present experiment is supported by recent observations that these three compounds have similar discriminative effects in both pigeons (Herling et al. 1981) and rats (Holtzman 1980), indicating similar pharmacological activity. In man, large doses of cyclazocine are frequently identified as barbiturates and produce effects including sedation and disorientation that are often associated with barbiturate administration (Jasinski 1977). Given these similarities in the effects of cyclazocine, dextrorphan, and ketamine, perhaps each of these compounds shares a component of action that is also shared by barbiturates.

The variability observed in the generalization of various drugs to pentobarbital suggests that the discriminative effects of pentobarbital may not be based on a single action of the drug, but rather on a complex of several properties (cf. Barry and Krimmer 1977, 1978b). In the present study, individual animals differed with respect to which particular drugs produced responding similar to that occasioned by the training drug. It is possible that this individual variation occurred because responding in different monkeys was controlled by different aspects of the stimulus complex produced by the training or test drugs. A test drug such as diazepam may mimic most of the effects of pentobarbital and accordingly would control responding in a manner similar to pentobarbital in virtually all subjects. A drug such as ketamine may mimic only some of the effects of pentobarbital, or may have additional actions, and thus would produce pentobarbital-appropriate responding only in subjects for which the common discriminative effects of pentobarbital and ketamine predominate. The finding that dextromethorphan, in contrast to its structural analogue dextrorphan, produced little or no pentobarbital-appropriate responding in the present experiment is of interest, since the similarity of discriminative effects noted above for ketamine, dextrorphan, and cyclazocine in rats does not extend to dextromethorphan (Holtzman 1980). Moreover, dextromethorphan, in contrast to either dextrorphan or ketamine, produces little or no drugappropriate responding in pigeons that are trained to discriminate pentobarbital from saline (Herling et al. 1980). Thus, dextromethorphan appears to lack a pentobarbital-like component of action that may be shared by dextrorphan, cyclazocine, and ketamine.

Acknowledgements. This research was supported by USPHS Grant DA 02230. The technical assistance of Kari L. George and the helpful comments of James H. Woods and Jonathan L. Katz on an earlier draft of this manuscript are gratefully acknowledged.

\section{References}

Barry H III (1974) Classification of drugs according to their discriminable effects in rats. Fed Proc 33:1814-1824
Barry H III, Krimmer EC (1977) Discriminable stimuli produced by alcohol and other CNS depressants. In: Lal H (ed) Discriminative stimulus properties of drugs. Plenum Press, New York, p 73

Barry H III, Krimmer EC (1978 a) Pharmacology of discriminative drug stimuli. In: Ho BT, Richards DW III, Chute DL (eds) Drug discrimination and state dependent learning. Academic Press, New York, p 3

Barry H III, Krimmer EC (1978b) Similarities and differences in discriminative stimulus effects of chlordiazepoxide, pentobarbital, ethanol, and other sedatives. In: Colpaert FC, Rosecrans JA (eds) Stimulus properties of drugs: Ten years of progress. Elsevier/North Holland Biomedical Press, Amsterdam, p 31

Breimer DD (1976) Pharmacokinetics of methohexitone following intravenous infusion in humans. Br J Anaesth 48:643-649

Colpaert FC, Desmedt LKC, Janssen PAJ (1976) Discriminative stimulus properties of benzodiazepines, barbiturates, and pharmacologically related drugs; relation to some intrinsic and anticonvulsant effects. Eur J Pharmacol 37:113-123

Hein DW, Young AM, Herling S, Woods JH (1981) Pharmacological analysis of the discriminative stimulus characteristics of ethylketazocine in the rhesus monkey. J Pharmacol Exp Ther 218:7-15

Herling S, Coale EH Jr, Hein DW, Winger G, Woods JH (1981) Similarity of the discriminative stimulus effects of ketamine, cyclazocine, and dextrorphan in the pigeon. Psychopharmacology $73: 286-291$

Herling S, Valentino RJ, Winger G (1980) Discriminative stimulus effects of pentobarbital in pigeons. Psychopharmacology 71:21-28

Holtzman SG (1980) Phencyclidine-like discriminative effects of opioids in the rat. J Pharmacol Exp Ther 214:614-619

Jarbe TUC (1976) Characteristics of pentobarbital discrimination in the gerbil: Transfer and antagonism. Psychopharmacology 49:33-40

Jarbe TUC, Ohlin GC (1979) Discriminative effects of combinations of $4^{9}$-tetrahydrocannabinol and pentobarbital in pigeons. Psychopharmacology 63:233-239

Jasinski DR (1977) Assessment of the abuse potentiality of morphinelike drugs (Methods used in man). In: Martin WR (ed) Drug addiction I: Morphine, sedative/hypnotic and alcohol dependence (Handbook of Experimental Pharmacology, vol 45/I). Springer, Berlin Heidelberg New York, p 197

Johansson JO, Jarbe TUC (1975) Antagonism of pentobarbital induced discrimination in the gerbil. Psychopharmacologia 41:225-228

Krimmer EC (1974) Drugs as discriminative stimuli. Dissertation Abst Internat $35: 4572-\mathrm{B}$

Krimmer EC, Barry H III (1979) Pentobarbital and chlordiazepoxide differentiated from each other and from nondrug. Comm Psychopharmacol 3:93-- 99

Leberer MR, Fowler SC (1977) Drug discrimination and generalization in pigeons. Pharmacol Biochem Behav 7:483-486

Overton DA (1966) State-dependent learning produced by depressant and atropine-like drugs. Psychopharmacologia 10:6-31

Overton DA (1975) A comparison of the discriminable CNS effects of ketamine, phencyclidine, and pentobarbital. Arch Int Pharmacodyn Ther 215:180-189

Sharpless SK (1970) Hypnotics and sedatives. I: The barbiturates. In: Goodman LS, Gilman A (eds) The pharmacological basis of therapeutics (Fourth edition). MacMillan Publishing Co, New York, $\mathrm{p} 98$

Trost JG, Ferraro DP (1974) Discrimination and generalization of drug stimuli in monkeys. In : Singh JM, Lal H (eds) Drug addiction, vol 3: Neurobiology and influences on behavior. Stratton, New York, $\mathrm{p} 223$

Winger G, Stitzer M, Woods JH (1975) Barbiturate-reinforced responding in rhesus monkeys: dose comparisons of compounds with different durations of action. J Pharmacol Exp Ther 195:505-514

Witkin JM, Carter RB, Dykstra LA (1980) Discriminative stimulus properties of $d$-amphetamine-pentobarbital combinations. Psychopharmacology $68: 269-276$

Received February 18, 1981; Final version October 19, 1981 